\title{
CCD Sky Monitoring and Burst Alert
}

\author{
René Hudec, Jan Soldán \\ Astronomical Institute, CZ-251 65 Ondřejov, Czech Republic
}

\begin{abstract}
Two proposals for detection of optical flashes related to $\gamma$-ray bursts are briefly described. The aim of the first proposal is to develop a reliable monitoring service at optical wavelengths, and the second proposal deals with an automatically working telescope with full remote control via Internet network.
\end{abstract}

\section{Optical Transient Monitor (OTM)}

The idea is to get very wide-field monitoring of the sky with still reasonable quality and parameters and also to concentrate on brighter flashes, but with a high background elimination level (Hudec \& Soldán 1994). Recent results (Hudec et al. 1994a,b, Hudec 1993) indicate that there are bright transients exceeding fifth magnitude (1 $\mathrm{s}$ duration assumed). We suggest a low-cost automatic operation experiment with good time resolution, medium sensitivity and angular resolution, and excellent background elimination. Previous experiments in this field have shown that the main problem of these investigations is the high noise (i.e., background caused by false triggers) to signal ratio. It is rather important to be able to eliminate reasonably all background and false triggers. We suggest to develop a double CCD camera with two independent paths equipped with different filters and to develop a special software excluding false triggers and triggers caused by satellites. The OTM system consists of the following:

(1) The classical IBM PC-386, 486 computer as a host computer for the Texas Instruments Parallel DSP TMS320C40 card.

(2) The internal AT add-in card with two TMS320C40 processors with 1 MByte of static memory and 32 MBytes of dynamic memory.

(3) Two or four CCD cameras with wide-field lenses.

The basic parameters of the proposed test device are as follows: Lens: widefield, $f=6 \mathrm{~mm}, 1: 1.6$. Focal detector: CCD camera ST-8 SBIG (Santa Barbara Instrument Group, Santa Barbara, CA). Area of the focal detector: $13.8 \times 9.2$ $\mathrm{mm}, 1534 \times 1020$ pixels $\left(9 \mu \mathrm{m}\right.$ square each). Field of view: less than $66^{\circ} \times 88^{\circ}$ or $5808 \mathrm{deg}^{2}$ (28\% of the hemisphere) Angular resolution: $0: 04-0.1$. Number of optical paths in one device: 2. Estimated limiting magnitude: 6 (expected; for 1 $\mathrm{s}$ flashes). Time resolution: approximately $40 \mathrm{~s}$ (1 $\mathrm{s}$ exposure, rest downloading and data processing) for two cameras and $\sim 20 \mathrm{~s}$ for four cameras in one device 
(the time resolution will increase up to $10(5) \mathrm{s}$ if either a smaller area of the chip will be digitized, or, alternatively, the pixels of the whole area will be binned.

\section{Robotic telescope}

The goal of the project is the development of an automatically operated telescope. A new generation of control software will be developed, allowing the fully robotic operation of the system i.e. remote control of the telescope via international data network Internet without any human participation. This will minimize the time delays between the detection of high-energy phenomena on satellites and corresponding optical ground-based observations. The main scientific goal is to use the robotic telescope described here in the BACODINE project which calculates the positions of celestial $\gamma$-ray bursts detected by the NASA Compton Gamma Ray Observatory (CGRO) and distributes these positions very rapidly to participating optical ground-based observatories. The robotic telescope will receive this positional burst information by means of the Internet network and will be automatically pointed to burst positions and the wide $\left(\geq 10^{\circ} \mathrm{FoV}\right)$ and narrow $\left(\leq 1^{\circ} \mathrm{FoV}\right) \mathrm{CCD}$ cameras will start observations.

The configuration of the project is based on the use of commercially available hardware components. The test configuration consists of newly developed Celestron Ultima $2000 \mathrm{PC}$-controlled mount with a $30 \mathrm{~cm}$ aperture telescope and a wide-field camera both with SBIG ST-8 CCD cameras, and PC-IBM 486. This enables to duplicate the system easily at other places at low cost. The corresponding software will be fully designed and developed (within $\sim 1 \mathrm{yr}$ ) and will guarantee a high level of automatization. The final goal is to establish a network of automated robotic telescopes on several sites.

An automatically operated telescope with remote control from any terminal of the Internet network can be obviously applied also in other fields of astronomy and astrophysics. The system can be used with little modifications also for other satellite astronomy projects where quasisimultaneous ground-based optical data are necessary, such as experiments WATCH/GRANAT, HETE, INTEGRAL etc.

Acknowledgement: We acknowledge support provided by the Grant Agency of the Czech Republic (grant No. 202-93-0890).

\section{References}

Hudec R., 1993, A\&A 270, 151

Hudec R., Soldán J., 1994, ApJS 92, 675

Hudec R., Dedoch A., Pravec P., Borovička J., 1994a, A\&A 284, 839

Hudec R., Pravec P., Borovička J., 1994b, A\&A 284, 499 